\title{
Scale dependence of subgrid-scale model coefficients: An a priori study
}

\author{
Elie Bou-Zeid, ${ }^{1,2, a)}$ Nikki Vercauteren, ${ }^{2}$ Marc B. Parlange ${ }^{2}$ and Charles Meneveau ${ }^{3}$ \\ ${ }^{1}$ Department of Civil and Environmental Engineering, Princeton University, \\ Princeton, New Jersey 08544, USA \\ ${ }^{2}$ School of Architecture, Civil and Environmental Engineering, \\ École Polytechnique Fédérale de Lausanne-EPFL, Lausanne CH-1015, Switzerland \\ ${ }^{3}$ Department of Mechanical Engineering and Center for Environmental and Applied Fluid Mechanics, \\ The Johns Hopkins University, Baltimore, Maryland 21218, USA
}

(Received 1 July 2008; accepted 4 September 2008; published online 26 November 2008)

\begin{abstract}
Dynamic subgrid-scale models require an a priori assumption about the variation in the model coefficients with filter scale. The standard dynamic model assumes independence of scale while the scale dependent model assumes power-law dependence. In this paper, we use field experimental data to investigate the dependence of model coefficients on filter scale for the Smagorinsky and the nonlinear models. The results indicate that the assumption of a power-law dependence, which is often used in scale dependent dynamic models, holds very well for the Smagorinsky model. For the nonlinear model, the power-law assumption seems less robust but still adequate. (C) 2008 American Institute of Physics. [DOI: 10.1063/1.2992192]
\end{abstract}

\section{BACKGROUND}

The dynamic approach introduced by Germano et al. ${ }^{1}$ represents a significant milestone in the development of generalized subgrid scale (SGS) turbulence models for large eddy simulations (LES). The approach computes an optimal model coefficient based on information from the smallest resolved scales in a simulation using the Germano identity. Although the approach was formulated for the Smagorinsky model, ${ }^{2}$ the Germano identity can be applied to other SGS models as well (see, for example, Armenio and Piomelli ${ }^{3}$ ). The traditional dynamic approach makes the assumption of scale invariance, i.e., that the coefficients do not depend on filter scale. The same coefficients determined from the smallest resolved scale are used for the SGS. This scale invariance assumption has been found to break down under various conditions where the filter cutoff scale falls near a transition scale rather than in the inertial subrange.,

To overcome this deficiency, scale dependent dynamic models have been formulated ${ }^{5-9}$ and are beginning to be implemented for various applications. ${ }^{10-13}$ As with the traditional dynamic models, the smallest resolved scales are used to obtain the model coefficient. However, scale dependent formulations also interrogate the smallest resolved scale about the variation of the model coefficient with filter scale. This is done by using two test filtering operations that yield the coefficient values at two resolved scales (the classic dynamic approach uses only one test filter scale). The information at the two test filter scales is then extrapolated to compute an optimal model coefficient that applies to the unresolved scales.

An assumption has to be made in the scale dependent formulations regarding the functional dependence of the SGS model coefficients on scale. A power-law functional depen-

\footnotetext{
${ }^{\mathrm{a}}$ Electronic mail: ebouzeid@princeton.edu. Telephone: +1-609-258-5429. Fax: +1-609-258-2799.
}

dence has been used in all previous scale dependent model implementations. Previously reported a priori tests of the scale dependent mode ${ }^{14}$ already suggested that it can accurately predict optimal Smagorinsky model coefficients for the velocity field (as determined by matching measured and modeled SGS TKE dissipations), while the scale invariant formulation underpredicted the coefficients. In addition, a posteriori tests also show that simulations with scale dependent dynamic models using the power-law dependence assumption perform better than scale invariant formulations $^{5,8}$ (producing velocity profiles and spectra that match theoretical and experimental results more accurately). However, no formal and direct testing of the assumed functional form for the scale dependence has been performed to date. Verifying the accuracy of the power-law dependence of SGS model coefficients on filter scale (for momentum, heat, and passive scalars) is the main goal of this study.

The scale dependent formulation is particularly relevant to LES of high-Reynolds number rough boundary layer flows encountered in geophysical applications. In such cases, there is no hope of resolving near-wall viscous processes; the height of the first grid points above the ground and the filter scale in near-wall regions are comparable to the local integral scale, i.e., outside of the inertial range. Moreover, accurate SGS modeling is of particular importance in such applications because the SGS dominate the overall fluxes near the ground. Hence, the tests to be presented in this paper use data relevant to atmospheric boundary layer flow and transport.

\section{EXPERIMENTAL DATA AND ANALYSIS}

The analysis is mainly based on measurements obtained during the Lake-Atmosphere Turbulent Exchange (LATEX) field campaign (August-October, 2006) over Lake Geneva, Switzerland. Wind velocity, temperature and humidity profiles were measured at $20 \mathrm{~Hz}$ using a vertical array of four 

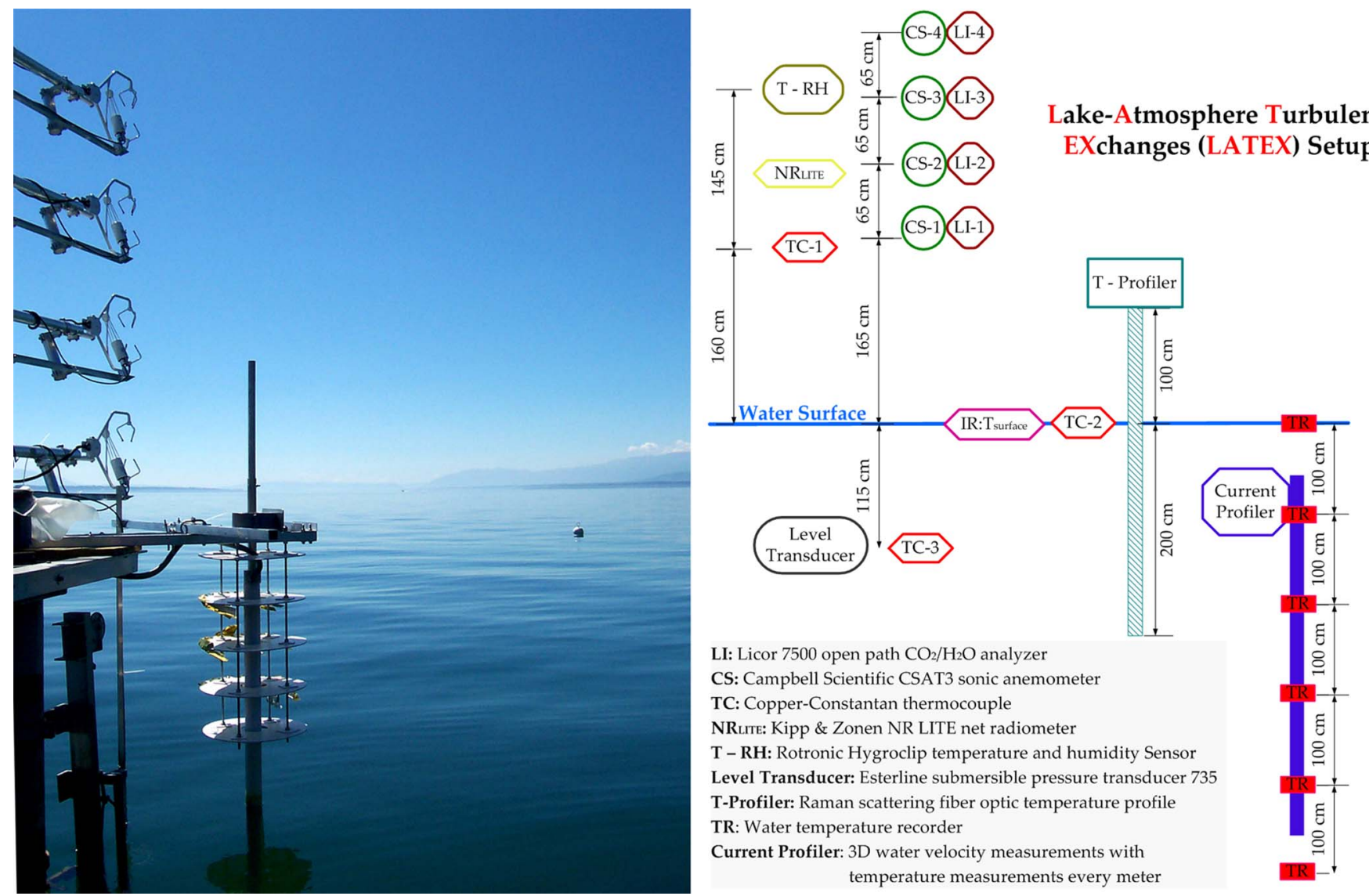

FIG. 1. (Color online) Setup of the vertical array over Lake Geneva during LATEX.

sonic anemometers (Campbell Scientific CSAT3) and open path gas analyzers (Licor-7500) (Fig. 1). The effective height of the measurements (middle point of the array) was $2.65 \mathrm{~m}$. This height is close enough to the surface for scale dependence of the coefficients to be significant (filter scale is of the same order of magnitude as the height above the surface, i.e. close to the production-range wavenumbers at that height). The details of the experiment and the a priori computations of the coefficients are presented in Vercauteren et al. ${ }^{15}$. LATEX data were mostly under neutral (no buoyancy) and convective (buoyant TKE production) atmospheric stability conditions; another data set is used in the last section of the paper to test the scale dependence under stable (buoyant TKE destruction) conditions.

The vertical array configuration allows the computation of all the SGS fluxes which are then used to compute a priori model coefficients by equating the measured and modeled SGS dissipations following

$$
\begin{aligned}
& c_{s}^{2}=\frac{-\left\langle\tau_{i j} \tilde{S}_{i j}\right\rangle}{2(\Delta)^{2}\left\langle\mid \tilde{S}_{i j} \tilde{S}_{i j}\right\rangle}, \\
& \operatorname{Pr}^{-1} c_{s}^{2}=\frac{-\left\langle q_{i}^{\text {heat }} \frac{\partial \tilde{T}}{\partial x_{i}}\right\rangle}{\left\langle\Delta^{2}|\widetilde{S}| \frac{\partial \widetilde{T}}{\partial x_{i}} \frac{\partial \widetilde{T}}{\partial x_{i}}\right\rangle}, \quad \operatorname{Sc}^{-1} c_{s}^{2}=\frac{-\left\langle q_{i}^{\mathrm{H}_{2}} \frac{\partial \frac{\partial \tilde{\rho_{\nu}}}{\partial x_{i}}}{\partial}{ }^{(1)}\right.}{\left\langle\Delta^{2}|\widetilde{S}| \frac{\partial \tilde{\rho_{\nu}}}{\partial x_{i}} \frac{\partial \tilde{\rho_{\nu}}}{\partial x_{i}}\right\rangle}
\end{aligned}
$$

for the Smagorinsky model and

$$
\begin{aligned}
c_{\mathrm{nl}}= & \frac{\left\langle\tau_{i j}{\tilde{S_{i j}}}\right\rangle}{\Delta^{2}\left\langle\frac{\partial \tilde{u_{i}}}{\partial x_{k}} \frac{\partial \tilde{u_{j}}}{\partial x_{k}} \tilde{S}_{i j}\right\rangle}, \\
c_{\mathrm{nl}}^{\text {heat }} & =\frac{\left\langle q_{i}^{\text {heat }} \frac{\partial \tilde{T}}{\partial x_{i}}\right\rangle}{\left\langle\Delta^{2} \frac{\partial \tilde{u_{i}}}{\partial x_{k}} \frac{\partial \tilde{T}}{\partial x_{k}} \frac{\partial \tilde{T}}{\partial x_{i}}\right\rangle}, \quad c_{\mathrm{nl}}^{\mathrm{H}_{2} \mathrm{O}}=\frac{\left\langle q_{i}^{\mathrm{H}_{2} \mathrm{O}} \frac{\partial \tilde{\rho_{\nu}}}{\partial x_{i}}\right\rangle}{\left\langle\Delta^{2} \frac{\partial \tilde{u_{i}}}{\partial x_{k}} \frac{\partial \tilde{\rho_{\nu}}}{\partial x_{k}} \frac{\partial \tilde{\rho_{\nu}}}{\partial x_{i}}\right\rangle}
\end{aligned}
$$

for the nonlinear model. ${ }^{16}$ In the above, $c_{s}^{2}, \operatorname{Pr}^{-1} c_{s}^{2}$, and $\mathrm{Sc}^{-1} c_{s}^{2}$ are the Smagorinsky model coefficients for momentum, heat, and water vapor, respectively; $c_{\mathrm{nl}}, c_{\mathrm{nl}}^{\text {heat }}$, and $c_{\mathrm{nl}}^{\mathrm{H}_{2} \mathrm{O}}$ are the nonlinear model coefficients for momentum, heat, and water vapor, respectively; $\tau_{i j}$ is the anisotropic part of the SGS stress tensor; $q_{i}^{\text {heat }}$ and $q_{i}^{\mathrm{H}_{2} \mathrm{O}}$ are the SGS fluxes of heat and water vapor, $\widetilde{S}_{i j}=0.5\left(\partial \widetilde{u}_{i} / \partial x_{j}+\partial \widetilde{u}_{j} / \partial x_{i}\right)$ is the resolved strain rate tensor, $\Delta$ is the filter scale, $T$ is temperature, $\rho_{v}$ is the concentration of water vapor in the air, $\mathbf{u}$ is the three-dimensional (3D) velocity vector; and the brackets denote averaging (in time for this study). However, only streamwise and vertical gradients can be computed with the LATEX setup; therefore, the two-dimensional (2D) surrogates of the strain rate tensors and SGS fluxes are used. ${ }^{15}$ This means that we only consider the 1-1, 3-3, and 1-3 components of the contractions in Eqs. (1) and (2) (1 and 3 
corresponding to the streamwise and vertical directions, respectively); for example, the Smagorinsky coefficient for heat is computed as

$$
\operatorname{Pr}^{-1} c_{s}^{2}=\frac{-\left\langle q_{1}^{\text {heat }} \frac{\partial \widetilde{T}}{\partial x_{1}}+q_{3}^{\text {heat }} \frac{\partial \widetilde{T}}{\partial x_{3}}\right\rangle}{\left\langle\Delta^{2}|\widetilde{S}|\left(\frac{\partial \widetilde{T}}{\partial x_{1}} \frac{\partial \widetilde{T}}{\partial x_{1}}+\frac{\partial \widetilde{T}}{\partial x_{3}} \frac{\partial \widetilde{T}}{\partial x_{3}}\right)\right\rangle} .
$$

The filtering operations (denoted by the tildes) are performed for the lower three and upper three probes separately to yield two filtered points and compute vertical gradients. 2D filtering is done using a box filter in the vertical direction and a Gaussian filter in the streamwise direction. Taylor's hypothesis is invoked to perform the streamwise filtering and to compute the streamwise gradients (see Vercauteren et al. ${ }^{15}$ for full details).

By changing the filter size $\Delta$, the model coefficient can be determined at different scales and the dependence of the coefficient on filter scale can be studied. We note that the 2D filtering in this analysis is performed in vertical planes, aligned with the streamwise direction; a comparison of vertical and horizontal filtering in a priori studies was presented in Higgins et al. ${ }^{17}$ We compute the coefficients for the basic square filter scale $\Delta=1.3 \mathrm{~m}$, and for two effective test filter scales $\Delta_{2}=\sqrt{2 \Delta}$ and $\Delta_{3}=2 \Delta$. Recall that the effective measurement height (height of the filter middle point) is $2.65 \mathrm{~m}$. The vertical size of the filter $\left(\Delta_{z}\right)$ is held constant at $1.3 \mathrm{~m}$ (due to setup constraints) and the streamwise size $\left(\Delta_{x}\right)$ is set to $1.3,2.6$, or $5.2 \mathrm{~m}$. The effective filter size is then computed as $\Delta=\left(\Delta_{x} \Delta_{z}\right)^{1 / 2}$, yielding the three filter scales of $\Delta$, $\sqrt{2 \Delta}$, and $2 \Delta$.

\section{RESULTS}

If the power-law assumption is made, i.e., a generic coefficient $C_{\Delta}=m \Delta^{\phi}$, it follows that

$$
C_{\alpha \Delta} / C_{\Delta}=\alpha^{\phi}=\beta=C_{\alpha^{2} \Delta} / C_{\alpha \Delta} .
$$

For our filter scales, this is equivalent to $\beta=C_{\Delta_{2}} / C_{\Delta}$ $=C_{\Delta_{3}} / C_{\Delta_{2}}$ (where $\beta$ follows the notation used in Ref. 5, 6, and 8). Note that these expressions (and scale dependent models) implicitly assume that a unique power law applies at the different scales and, hence, the power-law coefficients, $m$ and $\phi$, are themselves scale invariant.

To verify these assumptions, we can therefore plot $C_{\Delta_{2}} / C_{\Delta}$ versus $C_{\Delta_{3}} / C_{\Delta_{2}}$. Figure 2 depicts these two ratios for the Smagorinsky coefficient for momentum. The averaging operations required in Eqs. (1) and (2) are performed over 15 min chunks of data; tests with 1 min averages gave the same trends (with greater scatter of course). The collapse observed in Fig. 2 is very satisfactory, especially that a field experimental data set is being used where the effects of unsteadiness and measurement errors are typically higher than in laboratory settings or in direct numeric simulation data. The range of ratios displayed in the figure confirms that the coefficients are sensitive to filter scale and hence a verification of the scale dependence is indeed feasible; it also under-

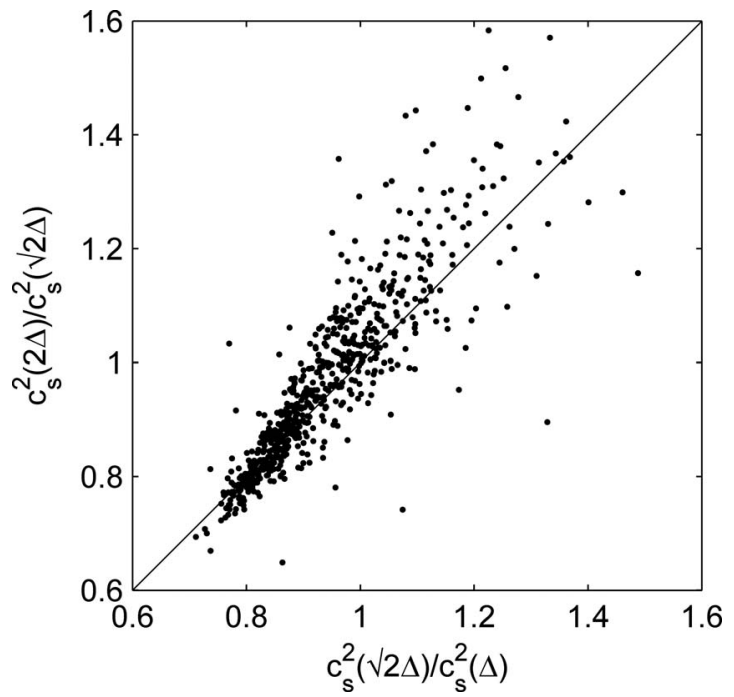

FIG. 2. Validation of the power-law dependence of the Smagorinsky coefficient on filter scale $\Delta$.

lines the importance of using scale dependent formulations since the coefficients are obviously not constant with scale.

Alternatively we can plot $C_{\Delta}$ versus $C_{\Delta_{2}}^{2} / C_{\Delta_{3}}$; this equality is actually directly used in some scale dependent model implementations ${ }^{8}$ to extrapolate the coefficient at the SGS scale $(\Delta)$ from its dynamically computed values at the test filter scales $\Delta_{2}$ and $\Delta_{3}$. Thus, it is more pertinent and will be used for the remainder of the paper. The computed value of $c_{s}^{2}(\Delta)$ versus its extrapolated value $c_{s}^{4}(\sqrt{ } 2 \Delta) / c_{s}^{2}(2 \Delta)$ is depicted in Fig. 3. Here, again, one can notice that the assumption of a power-law dependence of $c_{s}^{2}$ on $\Delta$ and the scale invariant extrapolation used in the scale dependent models hold very well. The linear regression fit (forced through the origin of the data: $y=b x$ ) yielded a slope $b$ of 0.986 with $R^{2}=0.96$.

We repeat the analysis for the SGS model coefficients for heat and water vapor. The results presented in Fig. 4 and 5 confirm that the power-law scale dependence assumption

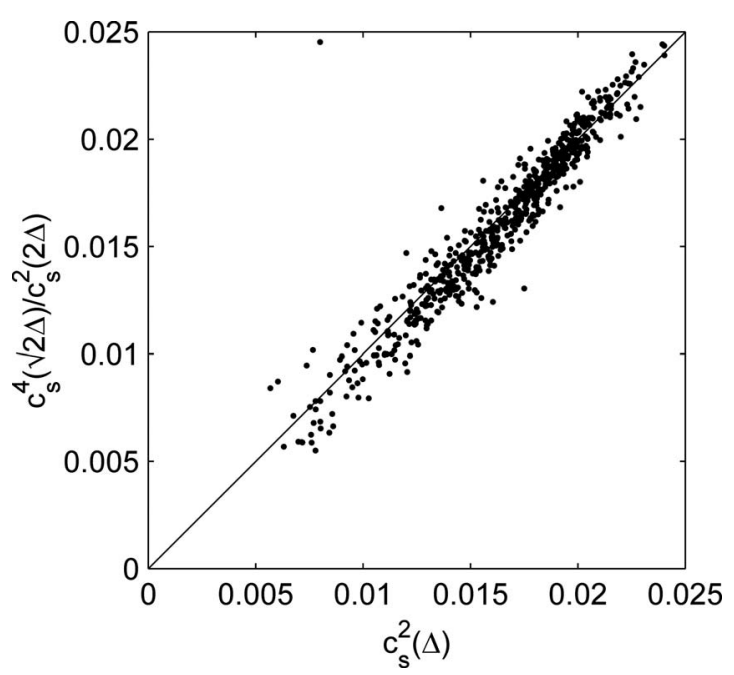

FIG. 3. Estimation of $c_{s}^{2}$ at a scale $\Delta$ from its values at scales $\sqrt{ } 2 \Delta$ and $2 \Delta$ using the power-law assumption. 


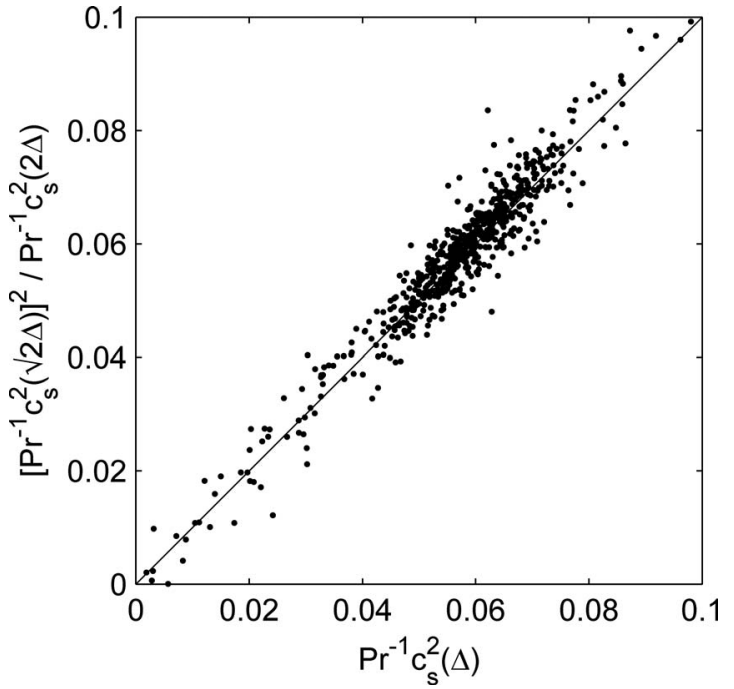

FIG. 4. Estimation of $\operatorname{Pr}^{-1} c_{s}^{2}$ at a scale $\Delta$ from its values at scales $\sqrt{ } 2 \Delta$ and $2 \Delta$ using the power-law assumption.

also holds very well for these coefficients and the extrapolation used in scale dependent models is therefore justified. The linear regression fit of the data $(y=b x)$ yielded slopes of about 1.018 and $R^{2}$ values of about 0.96 for both scalars. The results confirm that despite the significant variation in the value of the coefficients from one 15 minute average to the next (e.g., the coefficient varies between 0.006 and 0.025 ), the scale dependent assumption holds well for each of the 15 min averages individually (within the scatter observed in the plots).

The findings of the Smagorinsky model analysis can be extended to several other eddy-viscosity type models. For example, the Wong-Lilly model ${ }^{18}$ is a simple eddy viscosity model that proposes to compute the SGS eddy viscosity based on Kolmogorov scaling as

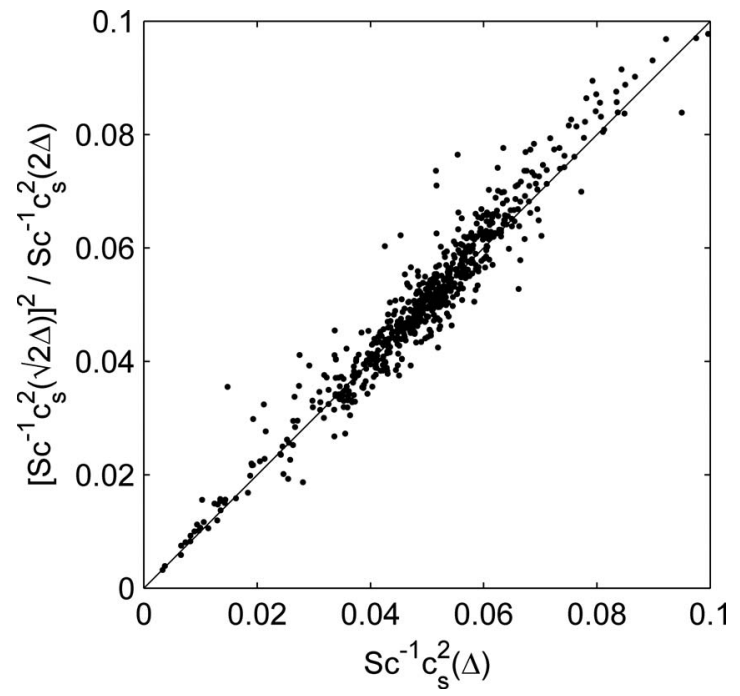

FIG. 5. Estimation of $\mathrm{Sc}^{-1} c_{s}^{2}$ at a scale $\Delta$ from its values at scales $\sqrt{ } 2 \Delta$ and $2 \Delta$ using the power-law assumption.

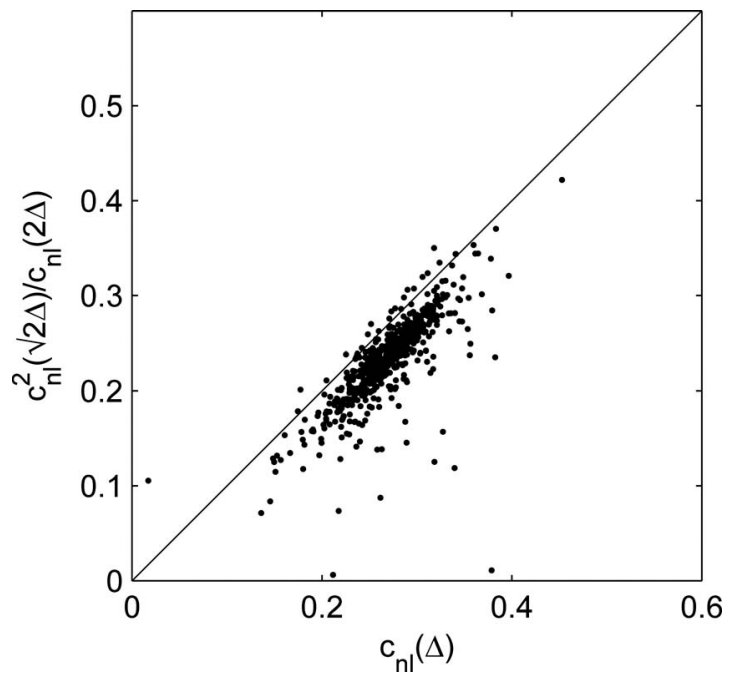

FIG. 6. Estimation of $c_{\mathrm{nl}}$ at a scale $\Delta$ from its values at scales $\sqrt{ } 2 \Delta$ and $2 \Delta$ using the power-law assumption.

$$
v_{\mathrm{SGS}}=C^{2 / 3} \varepsilon^{1 / 3} \Delta^{4 / 3}=C_{\varepsilon} \Delta^{4 / 3},
$$

where $C$ is the relevant dimensionless model coefficient, $\varepsilon$ is the (unknown) TKE dissipation rate, and $C_{\varepsilon}=C^{2 / 3} \varepsilon^{1 / 3}$ is the new model parameter that includes the dissipation rate. The parameter $C_{\varepsilon}$ is expected to become scale dependent when the filter scale is outside of the inertial subrange and Kolmogorov scaling (constant dissipation) is not applicable. In fact, since the Wong-Lilly parameter is dimensional, one may, in principle, expect higher sensitivity to filter scale than with the nondimensional Smagorinsky coefficients. Tests with LATEX data indeed show that the parameter $\beta$ in Eq. (4), when implemented for the Wong-Lilly model, varies approximately between 0.5 and 1.5 (compared to a range of $0.7-1.4$ for the Smagorinsky coefficient). Otherwise, the results are quite similar to those of the Smagorinsky model; in particular, the power-law assumption is well verified.

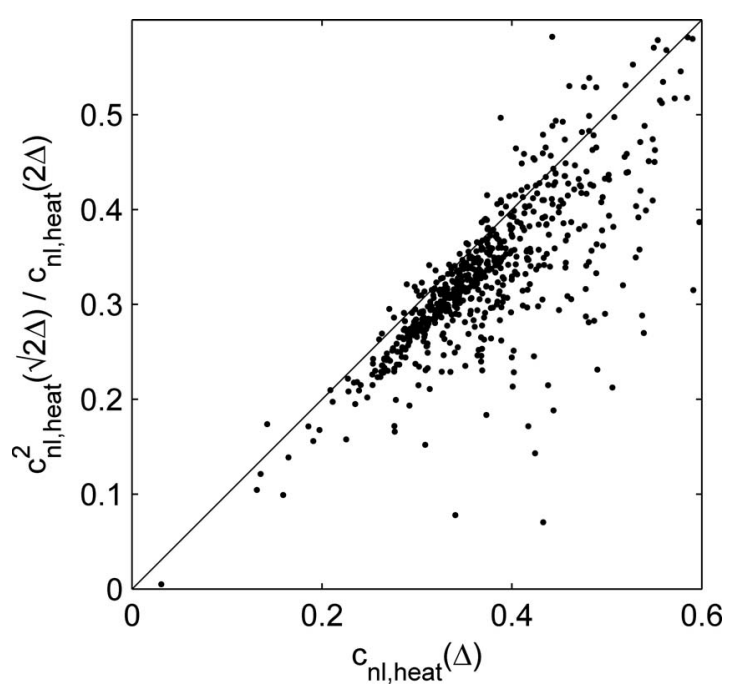

FIG. 7. Estimation of $c_{\mathrm{nl}}^{\text {heat }}$ at a scale $\Delta$ from its values at scales $\sqrt{ } 2 \Delta$ and $2 \Delta$ using the power-law assumption. 


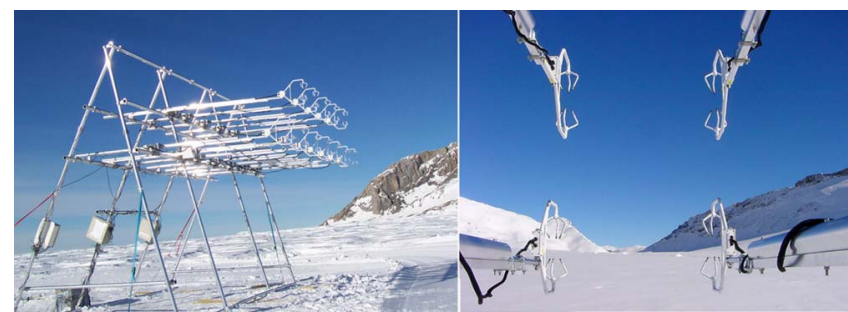

FIG. 8. (Color online) Side view of the SnoHATS setup of sonics arrays (left) and upwind fetch (right).

Next we consider the nonlinear model. As depicted in Fig. 6 for momentum and Fig. 7 for heat, the power-law dependence of the coefficient on filter scale is not as robust as with the Smagorinsky model. For water vapor, not shown here, the trend was very similar to that of heat. The slope of the linear regression fit for $c_{\mathrm{nl}}$ was about $0.88\left(R^{2}=0.87\right)$ and for $c_{\mathrm{nl}}^{\text {heat }}$ about $0.91\left(R^{2}=0.81\right)$. This indicates that the extrapolation of the SGS model coefficient based on the powerlaw assumption would underestimate the coefficient by about $10 \%$.

\section{EXTENSION TO STABLE CONDITIONS}

As previously mentioned, the LATEX experiment data tested here were mostly under neutral (no buoyancy) and convective (buoyant TKE production) atmospheric stability conditions. The stability is measured through the parameter $\Delta / L_{\mathrm{MO}}$, with $L_{\mathrm{MO}}$ being the Obukhov length

$$
L_{\mathrm{MO}}=\frac{-u_{*}^{3}}{\kappa \frac{g}{T_{v}} \overline{w^{\prime} T_{v}^{\prime}}} .
$$

In the above equation, $u_{*}$ is the friction velocity, $\kappa=0.45$ is the von Karman constant, $g=9.81 \mathrm{~m} / \mathrm{s}^{2}$ is the gravitational acceleration, $T_{v}$ is the virtual temperature, the prime denotes the turbulent part of a variable, and the overbar denotes Reynolds averaging. Since LATEX data were virtually always neutral or convective $\left(-20<\Delta / L_{\mathrm{MO}}<0\right)$, tests of the powerlaw scale dependence assumption under stable conditions were performed using another data set. The stable data was collected over a glacier where the snow cover ensured long periods of stable atmospheric stratification $\left(0<\Delta / L_{\mathrm{MO}}\right.$ $<10)$. The data are from the snow horizontal array turbulence study (SnoHATS) (Ref. 19) field experimental campaign performed over the extensive "Plaine-Morte" glacier in the Swiss Alps (7.5178 E, 46.3863 N, $2750 \mathrm{~m}$ elevation) from 2 February to 19 April 2006. Two horizontal arrays of vertically separated 3D sonic anemometers (Campbell Scientific CSAT3) were measuring wind and temperature at $20 \mathrm{~Hz}$ (Fig. 8) and allowed 2D filtering and computation of the full 3D gradients. The effective height of the measurement varied between 2.82 and $0.62 \mathrm{~m}$ due to snow accumulation during the experiment. Smagorinsky model coefficients are computed using all the terms of Equation (1) (3D gradients are available) with a basic square filter scale $\Delta=3.2 \mathrm{~m}$ (analysis with a basic filter scale $\Delta=2.25 \mathrm{~m}$ gave similar results). Box filtering is performed in the cross

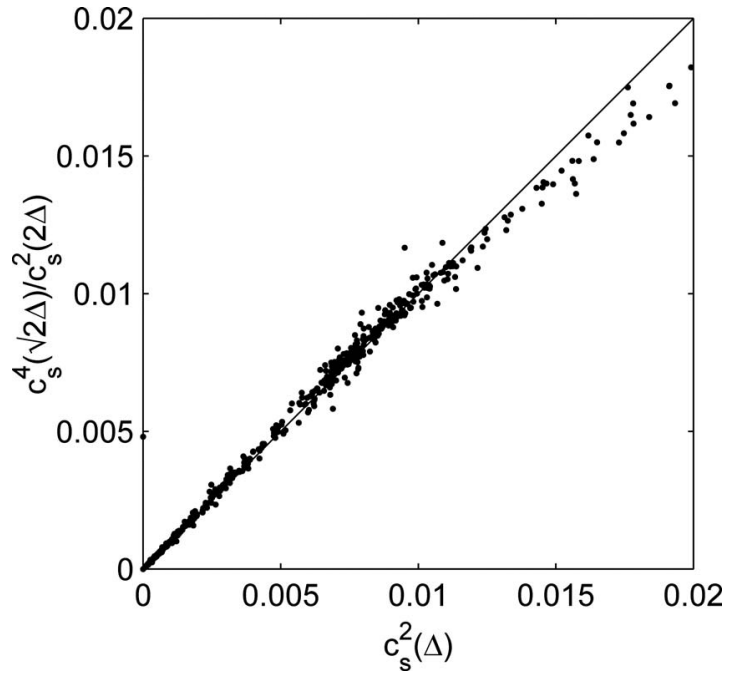

FIG. 9. Estimation of $c_{s}^{2}$ at a scale $\Delta$ from its values at scales $\sqrt{2 \Delta}$ and $2 \Delta$ using the power-law assumption under stable atmospheric conditions.

stream direction and Gaussian filtering is used in the streamwise direction by invoking Taylor's hypothesis. However, the 2D filter here is horizontal, as opposed to the vertical 2D filter used in LATEX.

Under stable conditions, buoyancy acts in the vertical direction to damp turbulence and mixing; therefore, a higher degree of anisotropy is expected. The reduction in the integral scale will also reduce the span of the inertial subrange, making the scale-invariance assumption even less robust and a scale dependent approach more indispensable. For the Smagorinsky model coefficients, previous studies observe a reduction in the coefficient magnitude as stability increases. ${ }^{20}$ The question is then whether stability will also affect the applicability of the power-law assumption for scale dependent models. Again previous studies reveal that the scale dependent formulation using a power-law assumption can better predict optimal model coefficients ${ }^{14}$ and perform better in a posteriori simulation of the diurnal cycle ${ }^{21}$ compared to a scale invariant formulation. SnoHATS data analysis (Fig. 9 for $c_{s}^{2}$ and Fig. 10 for $c_{s}^{2} / \operatorname{Pr}$ ) confirms that the power-law scale dependence assumption is still valid under stable condition. In fact, the data collapse seems slightly better than for the neutral and convective data from LATEX $\left(R^{2}\right.$ values of 0.99 for both momentum and heat compared to about 0.96 for LATEX); though this could be due to the availability of the full 3D gradients in SnoHATS (while in LATEX only the 2D surrogates are used) or to the longer averaging for SnoHATS (30 min) compared to LATEX (15 min). Longer averaging times are usually used under stable conditions to ensure statistical convergence of the mean turbulent fluxes. The linear fits $(y=b x)$ for the stable data of SnoHATS still give an accuracy of about $2 \%$ ( $b=0.986$ for momentum and 1.018 for heat), similar to the accuracy for LATEX data.

Results not shown here testing the nonlinear model using SnoHATS data display trends similar to the ones observed with LATEX: The applicability of the power-law scale dependence was less robust yielding $R^{2}=0.94$ and a fit slope 


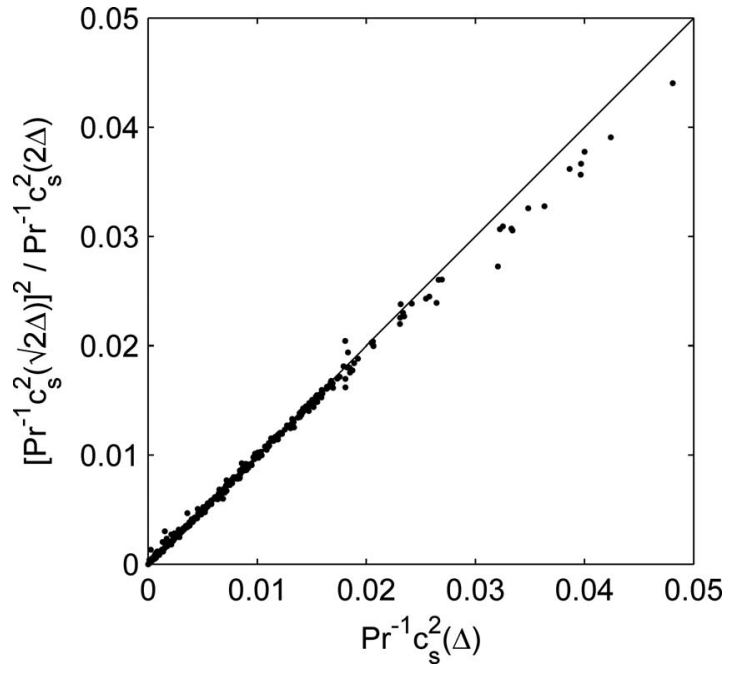

FIG. 10. Estimation of $\operatorname{Pr}^{-1} c_{s}^{2}$ at a scale $\Delta$ from its values at scales $\sqrt{2} \Delta$ and $2 \Delta$ using the power-law assumption under stable atmospheric conditions.

$b=0.91$ (again yielding about $10 \%$ underestimation of the model coefficient).

Of course, present conclusions are valid for the levels of stratification tested from the data $\left(\Delta / L_{\mathrm{MO}}<10\right)$. Stronger stratification could possibly lead to different results. Also, at strong stratification, differences may develop between the conclusions drawn from a priori and a posteriori tests. For instance, in LES of a daily cycle using the scale dependent dynamic model, Kumar et al. ${ }^{22}$ found that for $\Delta / L_{\mathrm{MO}}>4$, numerical instabilities developed.

\section{CONCLUSION}

This paper was aimed at the verification of the assumption that the SGS scale model coefficients vary with filter scale following a power law. Up until now, this assumption has been used in scale dependent dynamic subgrid models without direct a priori verification. Data from two field experiment were used: the LATEX experiment with mostly neutral and convective atmospheric conditions and SnoHATS experiment with mostly stable atmospheric conditions. The results indicate that the assumption holds very well for the coefficients of the Smagorinsky model for momentum, heat, and water vapor, under all atmospheric stabilities. For the nonlinear model, the assumption was less robust; however, the nonlinear coefficient could still be extrapolated based on the power-law assumption, albeit with an underestimation of about $10 \%$

\section{ACKNOWLEDGMENTS}

The authors would like to thank the Swiss National Science Foundation for its support for this work through Grant No. 200021-107910 and through the National Competence Center in Research on Mobile Information and Communication Systems (NCCR-MICS) under Grant No. 5005-67322.
C. Meneveau is supported by the US National Science Foundation under grant EAR-0609690.

${ }^{1}$ M. Germano, U. Piomelli, P. Moin, and W. H. Cabot, "A dynamic subgridscale eddy viscosity model," Phys. Fluids A 3, 1760 (1991).

${ }^{2}$ J. Smagorinsky, "General circulation experiments with the primitive equations: I. the basic experiment," Mon. Weather Rev. 91, 99 (1963).

${ }^{3}$ V. Armenio and U. Piomelli, "A Lagrangian mixed subgrid-scale model in generalized coordinates," Flow, Turbul. Combust. 65, 51 (2000).

${ }^{4}$ C. Meneveau and J. Katz, "Scale-invariance and turbulence models for large-eddy simulation," Annu. Rev. Fluid Mech. 32, 1 (2000).

${ }^{5}$ F. Porte-Agel, C. Meneveau, and M. B. Parlange, "A scale-dependent dynamic model for large-eddy simulation: Application to a neutral atmospheric boundary layer," J. Fluid Mech. 415, 261 (2000).

${ }^{6}$ F. Porte-Agel, "A scale-dependent dynamic model for scalar transport in large-eddy simulations of the atmospheric boundary layer," BoundaryLayer Meteorol. 112, 81 (2004).

${ }^{7}$ E. Bou-Zeid, C. Meneveau, and M. B. Parlange, "Large-eddy simulation of neutral atmospheric boundary layer flow over heterogeneous surfaces: Blending height and effective surface roughness," Water Resour. Res. 40, W02505, DOI:10.1029/2003WR002475 (2004).

${ }^{8}$ E. Bou-Zeid, C. Meneveau, and M. B. Parlange, "A scale-dependent Lagrangian dynamic model for large eddy simulation of complex turbulent flows," Phys. Fluids 17, 025105 (2005).

${ }^{9}$ R. Stoll and F. Porte-Agel, "Dynamic subgrid-scale models for momentum and scalar fluxes in large-eddy simulations of neutrally stratified atmospheric boundary layers over heterogeneous terrain," Water Resour. Res. 42, W01409, DOI:10.1029/2005WR003989 (2006).

${ }^{10}$ H. Y. Huang, B. Stevens, and S. A. Margulis, "Application of dynamic subgrid-scale models for large-eddy simulation of the daytime convective boundary layer over heterogeneous surfaces," Boundary-Layer Meteorol. 126, 327 (2008).

${ }^{11} \mathrm{~S}$. Radhakrishnan and U. Piomelli, "Large-eddy simulation of oscillating boundary layers: Model comparison and validation," J. Geophys. Res. 113, C02022, DOI: 10.1029/2007JC004518 (2008)

${ }^{12}$ W. S. Yue, M. B. Parlange, C. Meneveau, W. Zhu, R. van Hout, and J. Katz, "Large-eddy simulation of plant canopy flows using plant-scale representation," Boundary-Layer Meteorol. 124, 183 (2007).

${ }^{13}$ E. Bou-Zeid, M. B. Parlange, and C. Meneveau, "On the parameterization of surface roughness at regional scales," J. Atmos. Sci. 64, 216 (2007).

${ }^{14} \mathrm{~J}$. Kleissl, M. B. Parlange, and C. Meneveau, "Field experimental study of dynamic Smagorinsky models in the atmospheric surface layer," J. Atmos. Sci. 61, 2296 (2004).

${ }^{15}$ N. Vercauteren, E. Bou-Zeid, M. B. Parlange, U. Lemmin, H. Huwald, J. Selker, and C. Meneveau, "Subgrid-scale dynamics of water vapour, heat, and momentum over a lake," Boundary-Layer Meteorol. 128, 205 (2008).

${ }^{16}$ R. A. Clark, J. H. Ferziger, and W. C. Reynolds, "Evaluation of sub-gridscale models using an accurately simulated turbulent-flow," J. Fluid Mech. 91, 1 (1979).

${ }^{17}$ C. W. Higgins, C. Meneveau, and M. B. Parlange, "The effect of filter dimension on the subgrid-scale stress, heat flux, and tensor alignments in the atmospheric surface layer," J. Atmos. Ocean. Technol. 24, 360 (2007).

${ }^{18}$ V. C. Wong and D. K. Lilly, "A comparison of 2 dynamic subgrid closure methods for turbulent thermal-convection," Phys. Fluids 6, 1016 (1994).

${ }^{19}$ M. B. Parlange, E. Bou-Zeid, H. Huwald, M. Chamecki, and C. Meneveau, "SNOHATS: Stratified atmospheric turbulence over snow surfaces," in Advances in Turbulence XI: Proceedings of the 11th EUROMECH European Turbulence Conference, 25-28 June 2007, Porto, Portugal (Springer, Berlin, 2007), p. 520.

${ }^{20}$ J. Kleissl, C. Meneveau, and M. B. Parlange, "On the magnitude and variability of subgrid-scale eddy-diffusion coefficients in the atmospheric surface layer," J. Atmos. Sci. 60, 2372 (2003).

${ }^{21}$ J. Kleissl, V. Kumar, C. Meneveau, and M. B. Parlange, "Numerical study of dynamic Smagorinsky models in large-eddy simulation of the atmospheric boundary layer: Validation in stable and unstable conditions," Water Resour. Res. 42, W06D10, DOI:10.1029/2005WR004685 (2006).

${ }^{22}$ V. Kumar, J. Kleissl, C. Meneveau, and M. B. Parlange, "Large-eddy simulation of a diurnal cycle of the atmospheric boundary layer: Atmospheric stability and scaling issues," Water Resour. Res. 42, W06D09, DOI:10.1029/2005WR004651 (2006). 